\title{
1 Effects of reward history on decision-making and movement vigor
}

\author{
Shruthi Sukumar ${ }^{1}$, Reza Shadmehr ${ }^{3}$, Alaa A. Ahmed ${ }^{2}$ \\ Department of Computer Science, University of Colorado Boulder ${ }^{1}$ \\ Department of Mechanical Engineering, University of Colorado Boulder ${ }^{2}$ \\ Department of Biomedical Engineering, Johns Hopkins University ${ }^{3}$
}

Abstract

During foraging, animals decide how long to stay and harvest reward, and then abandon that site and travel with a certain speed to the next reward opportunity. One aspect of this behavior involves decision-making, while the other involves motor-control. A recent theory posits that control of decision-making and movements may be linked via a desire to maximize a single normative utility: the sum of all rewards acquired, minus all efforts expended, divided by time. If this is the case, then the history of rewards, and not just its immediate availability, should dictate how long one decides to stay and harvest reward, and how slowly one travels to the next opportunity. We tested this theory in a series of experiments in which humans used their hand to harvest tokens at a reward patch, and then used their arm to reach toward a subsequent opportunity. Following a history of poor rewards, people not only foraged for a longer period, but also moved slower to the next reward site. Thus, reward history had a consistent effect on both the decisionmaking process regarding when to abandon a reward site, and the motor control process regarding how fast to move to the next opportunity.

Financial support: This study was supported by grants from the National Institutes of Health (1R01NS096083 and 1R01NS078311). 


\section{Introduction}

Movements tend to be faster toward stimuli that promise a greater utility. For example, as the expectation of reward increases, peak speeds of both saccadic eye movements and reaching movements tend to increase (Haith et al., 2012; Rigoux \& Guigon, 2012; Shadmehr et al., 2016; Summerside et al., 2018; Takikawa et al., 2002; Thura et al., 2014). Similarly, as the effort cost of acquiring reward increases, speed of walking and reaching movements tends to decline (Gordon et al., 1994; Ralston, 1958; Schweighofer et al., 2015; J. Wang et al., 2021). Why does the prospect of greater reward invigorate movements?

One possibility is that by controlling movement vigor the brain is attempting to maximize an ecologically relevant utility: the reward that is expected at the successful conclusion of the act, minus the energetic expenditure required to perform that act, divided by time (Shadmehr et al., 2016). In this framework, faster movements are discouraged because they require greater energetic expenditure (Gordon et al., 1994; Ralston, 1958; Summerside et al., 2018). However, when there is reward at stake, faster movements save time, which reduces the discounted value of reward. When greater reward is at stake, the time that is saved by a faster movement may justify its energetic cost, and thus its vigor.

The mathematical formulation of this utility, reward minus effort divided by time, is the same as the one used by ecologists who study decision-making of animals during foraging (Stephens \& Krebs, 1986). In many of those studies, the objective is to understand why the animal decides to abandon a harvest site and move to another opportunity (Cowie, 1977; Richardson \& Verbeek, 1987). Optimal foraging theory operates on the notion that duration of harvest depends not only on the reward that is locally available, but also on the history of the subject, i.e., the past actions and their consequences (Stephens \& Krebs, 1986). For example, marginal value theorem, or MVT (Charnov, 1976), a method that finds foraging durations that maximize the global utility, predicts that if in the past the subject has enjoyed rich rewards, then it should abandon the current foraging site sooner, as compared to when the past has been one of scarcity. Indeed, animal studies have found a consistent effect of environment quality on behavioral parameters such harvesting duration and response latency (Cuthill et al., 1990; Niv et al., 2007; Perry et al., 2016; A. Y. Wang et al., 2013).

While studying saccadic eye movements and gaze behavior in humans, we suggested a generalized version of MVT to account for control of movement vigor (Yoon et al., 2018). The essence of the generalized theorem is that actions, whether they be in the context of harvesting, or moving between sites, should be controlled by a single global utility: the sum total of rewards and efforts, divided by time (Figure 1a). The generalized theory predicts that in environments in which rewards are plentiful or require little effort, the subject should not only abandon the reward site sooner, but also move with greater vigor toward the next opportunity (Figure 1b). That is, the rewarding outcomes and effort expenditures of past actions should have a consistent effect on both decision-making, and movement control.

While classical MVT has been empirically tested via examination of decision-making patterns (Constantino \& Daw, 2015; Cowie, 1977; Hayden et al., 2011; Krebs et al., 1974; Le Heron et al., 2020), the generalized predictions regarding the effects of history on motor control have yet to be tested. Here, we designed two foraging experiments in which we varied the history of reward and effort. Subjects used their hand to harvest tokens at a reward patch, and then used their arm to reach to the next patch. In each experiment, subjects performed these reach-and-harvest trials in various conditions: in rich environments where rewards were plentiful (or low effort expenditure associated with reaches between patches), or in poor environments where rewards were scarce (or high effort expenditure). Critically, in both environments we placed probe trials in which we controlled the availability of the immediate reward and the efforts required for attaining that reward. Theory predicted that when comparing behaviors in probe trials, the history of past actions, as 
reflected in the richness of the environment, should affect both reach vigor, and harvest duration. Indeed, following an experience of rich rewards, people not only reached with greater vigor in probe trials, they also abandoned those rewards sooner.

\section{Foraging Theory Predictions}

(a)

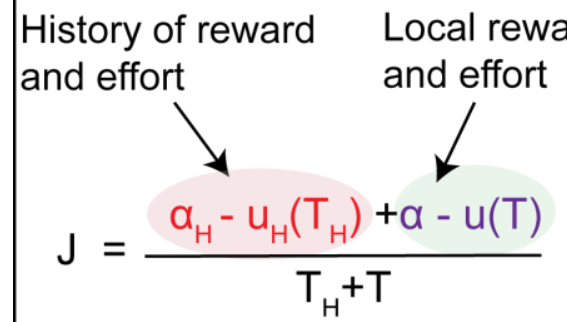

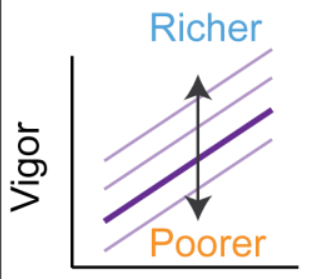

Reward

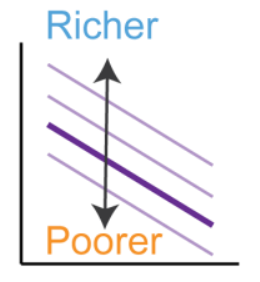

Effort

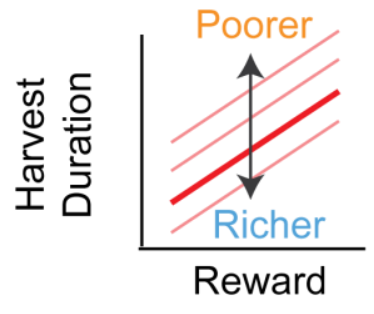

(b)
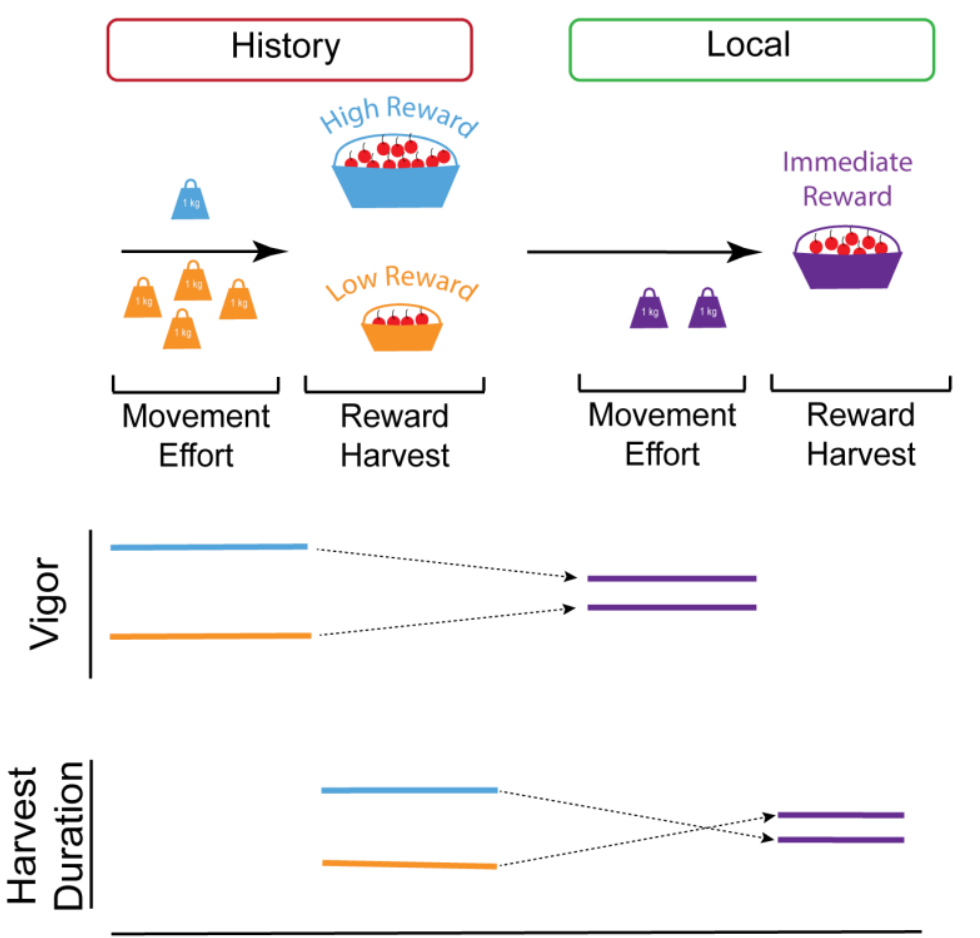

Time

Figure 1: $(a, b)$ Generalized MVT predictions for control of movement vigor and harvest duration (a) Richer environment with history of high reward or low effort will elicit higher vigor. But richer environments will also elicit lower harvest duration. (b) After a history of low effort or high reward, the vigor of the current movement will increase as compared to a history of high effort and low reward. Similarly, after a history of high reward and low effort, harvest duration will decrease as compared to history of low reward and high effort. Note that while environmental richness is positively correlated with vigor, it is negatively correlated with harvest duration, despite immediate reward increasing both.

\section{Results}

In a foraging task (Experiment 1, Figure 2c), subjects held a robotic manipulandum and used their hand to apply force pulses that produced tokens (virtual berries) at reward patches (the berries were exchanged into a monetary bonus at the end of the experiment). There were three patch types, indicated by their color, with differing reward rates (low, medium, and high, Figure 2d). As they stayed within a patch and continued to harvest, the number of berries that we delivered with each pulse decayed (Figure 2c, third panel), thus encouraging them to leave. Their decision to leave was aided by the fact that as they harvested, the location and reward rate of the next patch was displayed, making it possible for them to leave the current patch and reach to the next patch when they wished to. 
(a)

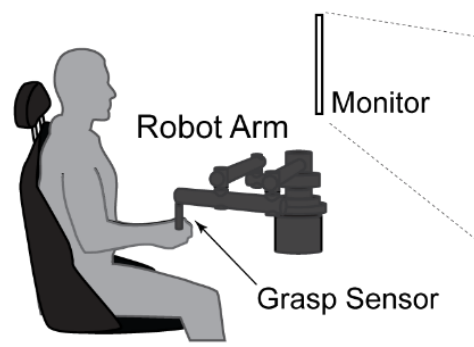

Experiment 1

(c)

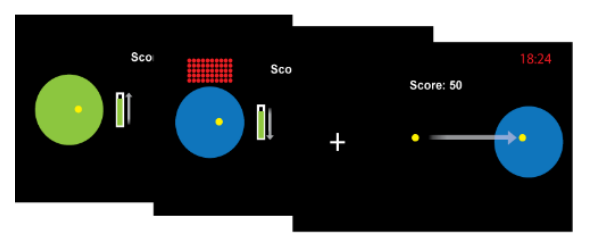

(e)

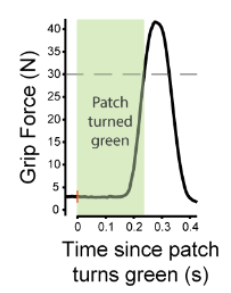

(f) (f)

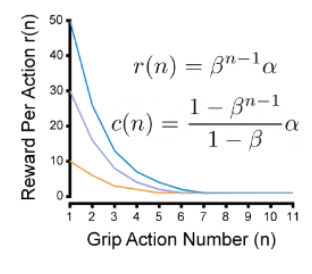

- Probe Trials (b)

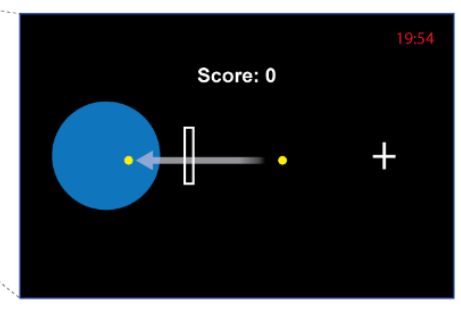

Experiment 2

(d)
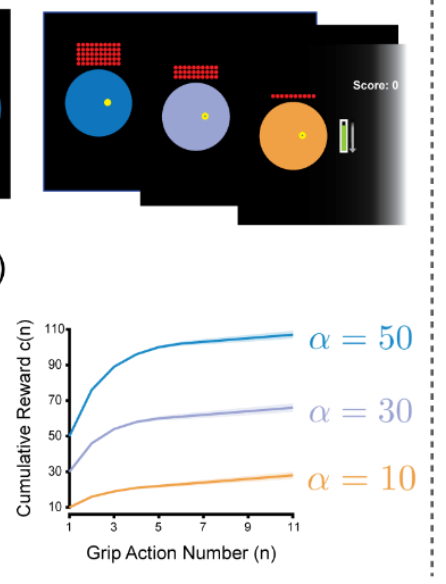

(j) (g)

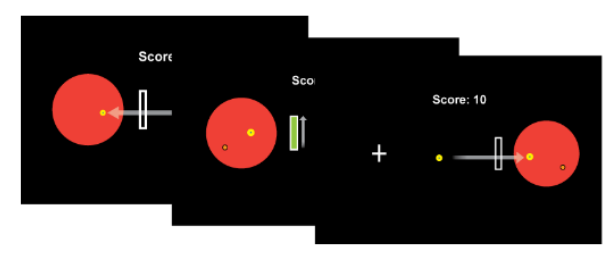

(h)

(i)

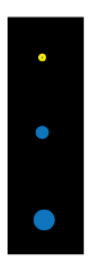

$0 \mathrm{~kg}$

$2 \mathrm{~kg}$

$3.5 \mathrm{~kg}$

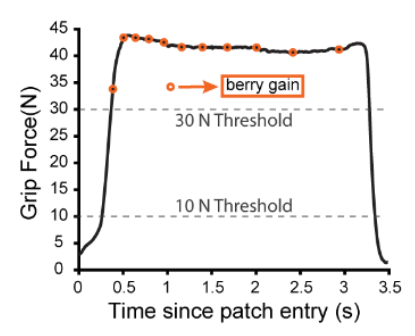

Trial

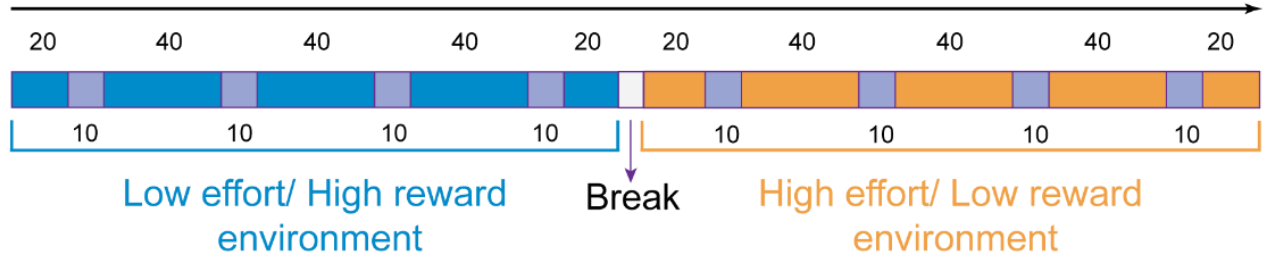

Figure 2: Experiment design. (a) Participants were seated in front of a computer monitor while grasping the handle of a robotic arm. The robot handle housed a sensor that measured grip force. (b) They used the robot to move the cursor and place it inside the target ("patch") to earn reward (berries). In Experiment 1, we varied the amount of reward at each patch. (c) Once in the patch, harvesting would commence by waiting for 1 second (green light), and then producing a force pulse. Each subsequent grip pulse had to wait for $1 \mathrm{sec}$ and was rewarded with an exponentially reduced number of berries. The number of berries collected was added to a running score, which was displayed on the screen. Subjects were free to stop harvesting and move to the next patch at any time. (d) The richness of each patch was cued by a specific color. (e) Example of a grip pulse resulting in berry harvest. (f) Different reward functions for the three patch types plotted wrt number of grips applied within the patch (left). Cumulative rewards for the three patch types as a function of grip number within patch (right). In Experiment 2, we varied the effort required to travel between patches. (g) A red 'patch' served as the cue, instructing the subject to move the cursor to collect reward. Once in the patch they increased grip force to a threshold (bar height) to commence harvesting. During harvesting, grip force had to be maintained above threshold $\left(F_{g}=30 \mathrm{~N}\right)$ to continue berry collection. Subjects could move out at any point to the next patch. (h) To modulate effort cost of travel between patches, we added a mass to the reaching movements, indicated by a circle that was proportionately sized to the mass and appeared over the cursor. (i) Example grip force profile; subjects had to hold force above $\left(F_{g}=30 N\right)$ to collect berries indicated by orange circles. (j) Protocol for both experiments. Each rectangle represents a contiguous block of trials with corresponding number of trials indicated. Note 
that for experiment 1 each environment included probe trials and was conducted for a fixed amount of time (20 minutes), with the remaining time displayed in the upper right corner of the screen.

The key variables were the length of time they decided to stay and harvest, and more importantly, the velocity with which they reached to the next reward patch. We hypothesized that these variables depended not only on the reward properties of the current patch, but also reward history. To control the reward history, the patches were organized so that the subjects initially experienced either a poor (e.g., most patches containing a small amount of reward, Figure $2 \mathrm{j}$ ) or a rich environment. In addition, in both environments subjects also encountered probe trials in which the immediate reward rate was kept constant (medium). These probe patches allowed us to measure the effect of past reward rate while controlling for the immediate reward. Subjects were given a fixed amount of time $(20 \mathrm{~min})$ to complete each environment (Figure $2 \mathrm{~h}$ ) and were familiarized with the various patches before the main experiment.

\section{History of high reward led to faster movements, and earlier abandonment of the reward patch}

Subjects reached faster toward patches that promised a greater immediate reward, as shown for a representative subject in Figure 3a, and across subjects in Figure $3 b\left(F(1,1104)=80.225, p<2 * 10^{-16}\right)$. However, when the promise of reward was equalized (probe trials), reach speed depended on the history of the subject: following a history of high reward trials, reach speed in probe trials was greater than following a history of poor reward trials [peak velocity in probe trials following a high reward environment as compared to the low reward environment, $F(1,264)=13.661, p=0.0002$ ] (Figures $3 \mathrm{~b}$ and $3 \mathrm{c}$ ). Thus, the history of reward affected reach vigor: reach velocity was higher in probe trials that followed a sequence of high reward experiences.

Once the hand entered a reward patch, the subjects produced a series of force pulses to collect berries (one pulse per set of berries). Figure $3 d$ provides an example of the force pulses, and the number of berries collected following each pulse (red dots atop each pulse). The raster in Figure 3e provides an example of harvest behavior in the rich and poor environments, as well as in the probe trials (same subject as in Figure 3a). As expected, the subject stayed longer in the high reward patch as compared to the low reward patch, a pattern that was consistent across subjects (harvest duration was longer in the high reward patch, Figure $3 \mathrm{~g} ; F(1,1104)=632.4, p<2 * 10^{-16}$ and more berries were collected; $F(1,1104)=10838, p<2 *$ $\left.10^{-16}\right)$. However, the length of harvest in probe trials depended on the history of the subject; they abandoned the harvest location sooner if the past was one of high rewards. Indeed, after a history of high reward the subjects chose to stay for a shorter period in the probe patch (Figure $3 \mathrm{~h}, F(1,264)=$ $37.134, p<3.8 * 10^{-9}$ ), and collected fewer berries (Figure 3i, $F(1,264)=63.077, p<5 * 10^{-14}$ ), as compared to the probe patch that followed low rewards. In other words, given the same amount of immediate reward (i.e., probe trials), if the history was one of high reward the subjects tended to abandon the current patch earlier, thus leaving with fewer berries.

As they harvested rewards, subjects could see the location of the next patch and whether it contained high, medium, or low rewards. This allowed us to examine whether the prospect of future rewards affected behavior at the current patch. We noticed a curious change in behavior in the trial just before the onset of the probe patch: during the final harvest in the high reward patch, subjects lingered longer than normal if the next patch was a probe trial (harvest duration, trial 19 vs. $20, t(13)=3.258, p=0.0062$ ). In other words, just before moving to a relatively poor patch (probe trial), subjects stayed longer than usual in the high reward patch. This transition from high reward patches to a probe patch also produced a relatively slow reaching movement (as compared to reaches to probes that were preceded by other probes, $t(13)=$ $-2.188, p=0.0475)$. Similarly, when the next patch contained a relatively greater amount of reward 
145 than the current one, harvest duration was shorter than normal (final harvest in the low reward environment 146 when the subsequent patch was probe, $t(13)=-3.53, p=0.0037)$. Thus, the harvest duration was longer 147 than usual if the promise of future reward was lower than the current rate.

148 In summary, immediate reward affected harvest duration and reach vigor: in the high reward environment 149 people lingered longer at each patch (as compared to low reward), and then reached with greater vigor 150 toward the next high reward patch. In addition, the history of reward also affected these variables: following 151 a low reward history, people lingered longer at the probe site, and then reached with reduced velocity to 152 their next opportunity. Thus, as theory had predicted, a history of high reward encouraged earlier 153 abandonment of the current reward patch, and faster movements toward the next patch. 
bioRxiv preprint doi: https://doi.org/10.1101/2021.07.22.453376; this version posted July 23, 2021. The copyright holder for this preprint (which was not certified by peer review) is the author/funder. All rights reserved. No reuse allowed without permission.

(a)

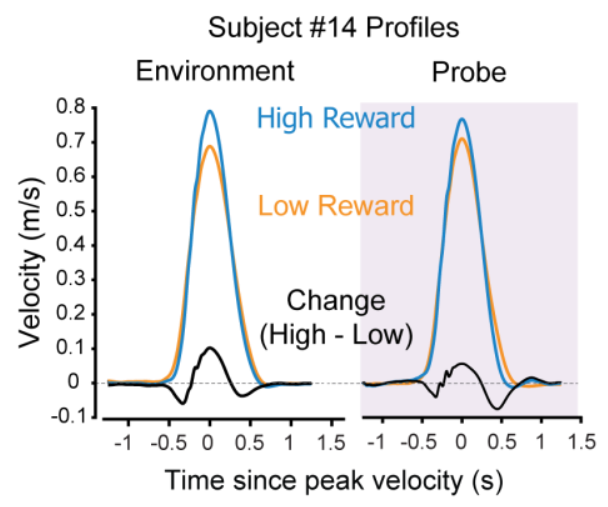

(d)

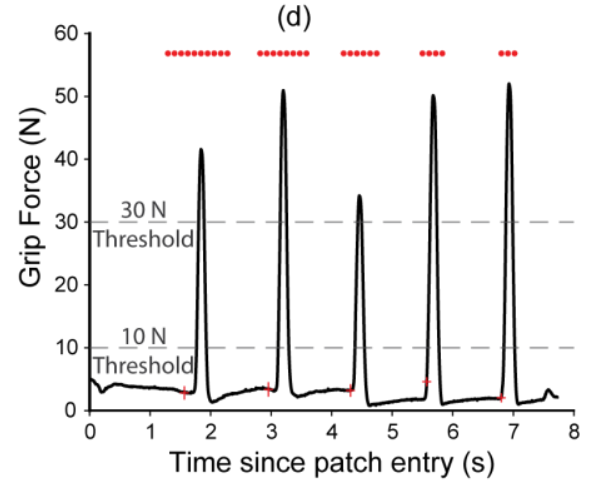

(f)

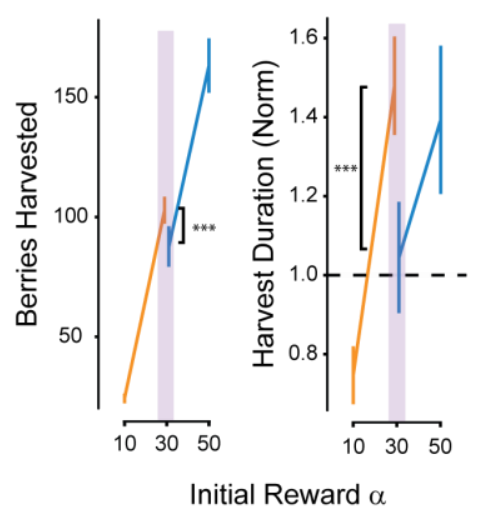

(b)

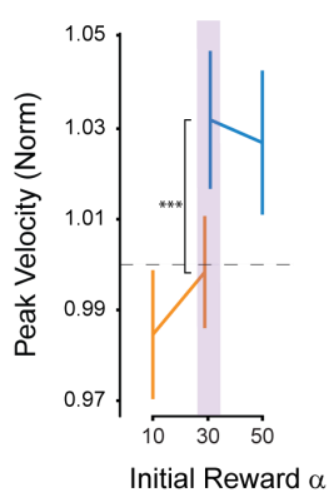

Low Reward $(\alpha=10)$

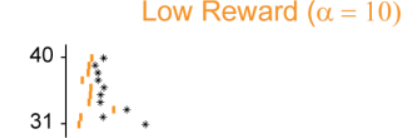

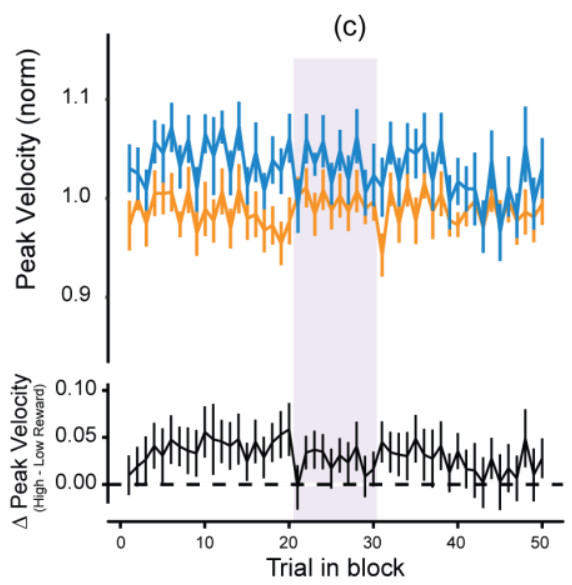

(e)

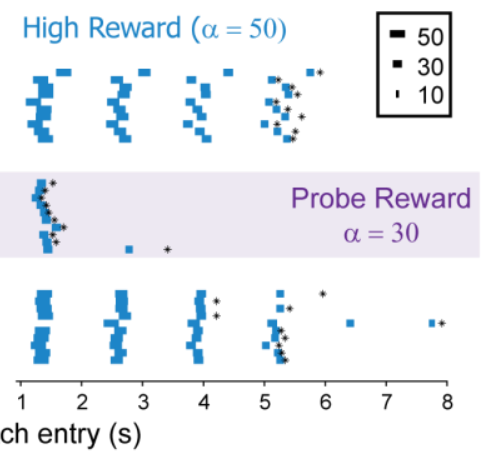

(h)

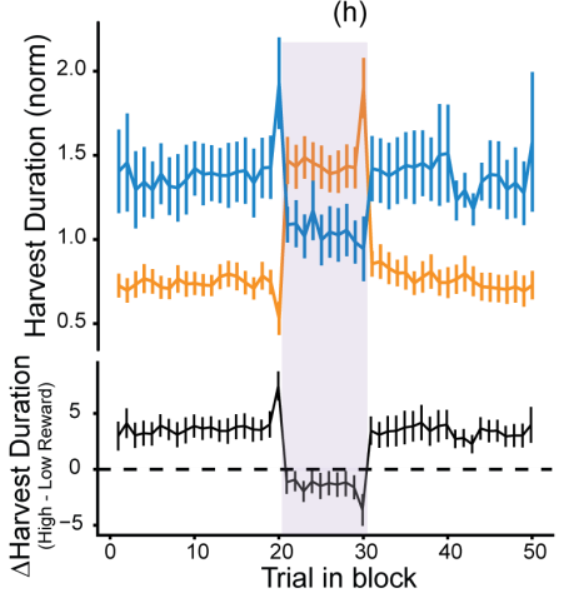

(i)

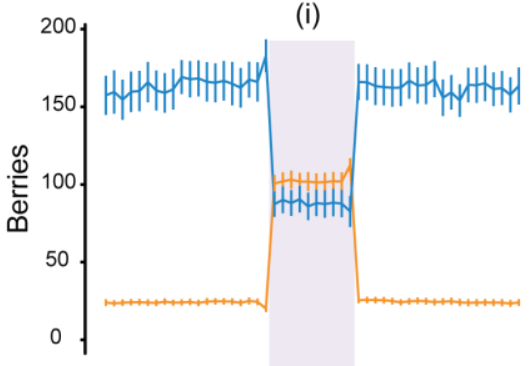

154

155

156

157

158

159

160

161

162

163

164

165

166
Figure 3: (a) Velocity profile averaged across trials belonging to each environment for a representative subject; probe trials are separated out for each environment. (b) Normalized peak velocity plotted with respect to reward in patch on the current trial; each point represents the average of the mean normalized peak velocity across all subjects. Subjects are faster in probe trials belonging to high reward environment $\left({ }^{*} p<0.05,{ }^{* * *} p<0.001\right)$. (c) Mean peak velocity (normalized to subject average) within a block of 50 trials, averaged across subjects, plotted as a function of trial for the low (blue) and high (yellow) effort environments. Corresponding difference $\triangle P V$ is plotted with respect to trial number within block right below. All error bars represent standard error of the mean. (d) A sample grip force profile for an example subject \#14 on a randomly selected trial to indicate the temporal course when inside a patch. In this case, subject is harvesting from a low reward patch; initial patch reward is 10 and successively decreases with grip actions. (e) Raster plot showing berries harvested in 30 representative trials per environment including for Subject \#14. Each row represents a trial. Annotated trials are probe trials with equivalent reward across environments. Each point represents a harvest with the thickness of the point represents the number of berries harvested. Time of departure from patch is indicated by the asterisk for corresponding trial. $(f, g)$ Berries harvested $(w)$ and harvest duration (normalized 
to subject average; f) plotted as a function of patch initial reward $\alpha$. Comparing metrics between the probe patches average between the two environments showed subjects left patches sooner after history of high reward. (h,i) Mean number of berries $(\mathrm{g})$ and mean harvest duration, normalized to subject average $(\mathrm{h})$ within a block of 50 trials, averaged across subjects, plotted as a function of trial for the low (blue) and high (yellow) effort environments. Corresponding difference is plotted with respect to trial number within block right below. All error bars represent standard error of the mean.

\section{A history of high effort expenditure encouraged slower reaching movements}

In experiment 2, we shifted our focus from history of reward to history of effort expenditure. We did this by simulating an added mass on the hand as the subjects reached between patches (via an accelerationdependent resistive force field). Like experiment 1, subjects foraged for discrete rewards by reaching towards them (Figure 2f). Unlike experiment 1, once at a patch they applied a constant grip force to obtain reward. As before, they were free to stop the harvest and leave for the next patch at any time (Figure $2 \mathrm{f}$, third panel). They experienced three added mass conditions over the course of the experiment which were cued through the appearance of the cursor (Figures $2 \mathrm{~g}$ ). In addition, they foraged in two environments: a high effort environment ( $3.5 \mathrm{~kg}$ mass) and a low effort environment ( $0 \mathrm{~kg}$ mass). In each environment, there were intermittent probe trials $(2 \mathrm{~kg}$ ), which allowed us to compare behavior between environments when the immediate effort requirements were the same, but history of effort differed.

As expected, subjects reached faster when carrying a small mass as compared to a large mass (effect of environment effort on peak velocity, as illustrated by data from a single subject in Figure 4a, and group data in Figure $\left.4 \mathrm{~b}\left[F(1,1420)=3828.4, p<2 * 10^{-16}\right]\right)$. More importantly, the history of effort affected their reaching movements: reach velocity was higher in probe trials when those trials were embedded in an environment in which effort costs were low (Figure $4 \mathrm{~b},\left[F(1,1420)=27.31, p<3.03 * 10^{-7}\right]$ ). Thus, as expected the subjects reached slower if the mass on the probe trial was greater than the trials that immediately preceded. However, this reach speed was faster if the probe trial was embedded in a low effort environment as compared to a high effort environment.

Interestingly, the effect of effort history on vigor was not uniform across all subjects (Figure 4d). Specifically, five of the 18 subjects presented a slight or strong increase in probe trial vigor in the high effort environment. One possible explanation for this is that, despite the available reward and reward rate being uniform in patches across both environments, subjects valued the reward in the high effort environment more by the virtue of having spent more effort to acquire it. This is addressed further in the Discussion section.

Upon entering a patch, subjects increased their grip force to the threshold required for harvesting. This threshold remained constant despite the varying effort requirements (i.e., masses) of the environment. Interestingly, subjects altered the rate at which they increased their grip force (grip ramp-up phase in Figure 5a) based on the effort history of the environment: in the probe trials that were placed in the high effort environment, the rate of increase in grip force tended to be slower.

Two examples of harvesting trials are shown in Figure 5b. This subject increased their grip force faster in the context of the low effort environment. We quantified this behavior by measuring the rate of force increase as well as the duration of time it took to ramp force from $10 \mathrm{~N}$ to the threshold of $30 \mathrm{~N}$. (Subjects were required to maintain their force below $10 \mathrm{~N}$ before they enter the patch thereby determining the lower threshold.) We found that on average, peak rate of force was higher in trials belonging to the low effort environment as opposed to the high effort environment [example subject Figure 5c; two-way repeated measures ANOVA, main effect of environment on peak force rate $F(1,264)=5.714, p=0.0175]$. When we focused on probe trials, we found that peak force rate in the context of the low effort environment was 
higher than in the context of the high effort environment [Figure 5d-e; two-way repeated measures ANOVA, effect of environment effort on probe trials, $F(1,1382)=17.442, p<0.001]$. When considering the correlated metric of grip ramp-up duration we see an interaction between environment effort and trial type [two-way repeated measures ANOVA on all trials reveals a slightly significant interaction between environment effort and trial type, $F(1,264)=3.851, p=0.05076]$. Focusing once again on just the probe trials we found a significant effect of environment effort on grip ramp-up duration [Figures $5 f-$ $\mathrm{g} ; F(1,1382)=8.909, p=0.00289]$. From Figures $4 \mathrm{~d}$ and $5 \mathrm{e}$, we notice that there is a significant overlap among subjects who had a higher peak force rate in the high effort environment and those who moved faster in the high effort environment. Specifically, subjects 6, 7,11 and 13 who have higher peak velocity in the high effort environment also grip faster in the high effort environment. This provides more evidence in support of the notion that these subjects view the high effort environment as better than the low effort environment.

Following the ramp-up phase, subjects harvested berries. In contrast to the grip ramp-up phase, we found no consistent effect of environment effort ( $p=0.690$ ) on number of berries collected (Figure 5 h), even when focusing on only the probe trials $(p=0.621)$. Finally, we focused on the behavior as the subjects ended the harvest period and reduced their grip force, termed giving-up phase (Figure 5a), or the duration that subjects waited in a patch gripping after collecting their last berry. Since the duration between consecutive berries increased with time spent in the patch, this metric was normalized to the time experienced waiting between the second-to-last and last berries. We found that this normalized giving-up time was modulated significantly by environment effort (Figure 5i [two-way repeated measures ANOVA, main effect of effort; $F(1,264)=5.437, p=0.0205]$ ). That is, in the high effort environment, the subjects waited longer before giving up. Importantly, we found no significant effect of environment $[F(1,1382)=2.088, p=0.149]$.

In summary, for identical immediate effort and reward opportunities, movement vigor tended to be modulated by the subject's history of experience. Following a history of high effort expenditure, people reached with reduced velocity in probe trials. Following a history of high effort, subjects did not significantly modulate the amount of reward that they harvested. However, they took longer to begin harvesting reward (grip ramp-up period) and waited longer before ending the harvest and moving on to the next patch (giving-up phase). 
(a)

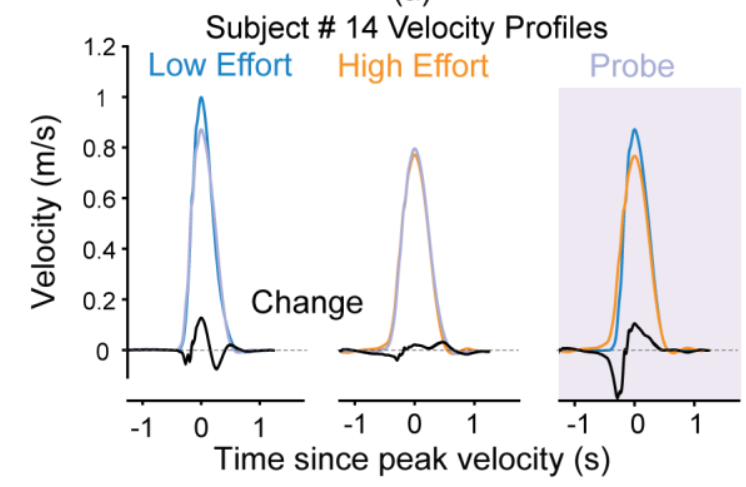

(c)

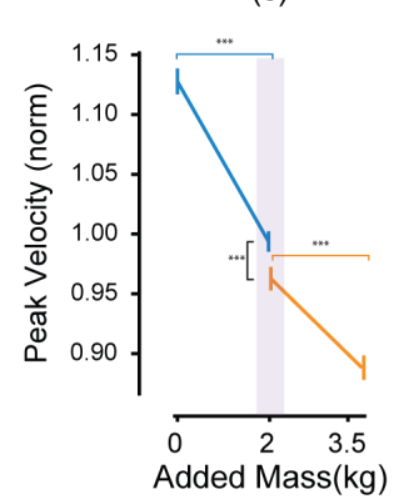

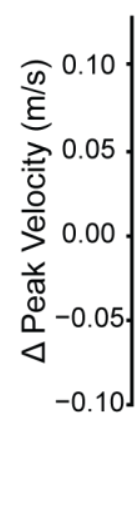
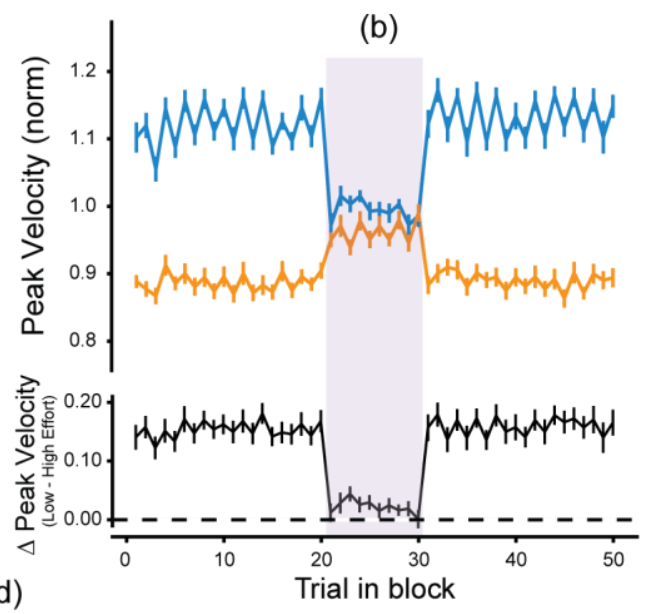

(d)

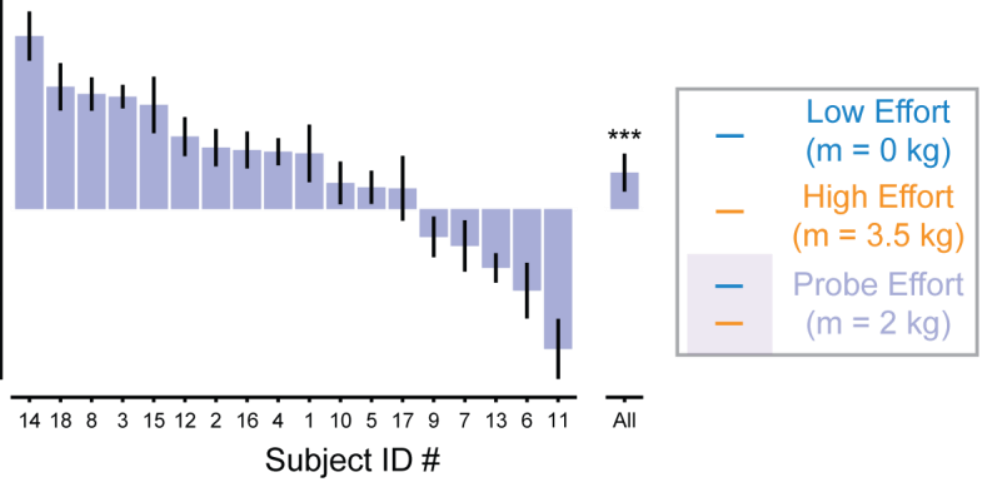

Figure 4: Vigor was influenced by history of effort. (a) Averaged velocity profiles for different mass conditions, Okg (blue), $2 \mathrm{~kg}$ (purple background) and $3.5 \mathrm{~kg}$ (yellow), for an exemplar subject 14. (b) Mean peak velocity (normalized to subject average) within a block of 50 trials, averaged across subjects, plotted as a function of trial for the low (blue) and high (yellow) effort environments. Corresponding difference $\triangle P V$ is plotted with respect to trial number within block right below. All error bars represent standard error of the mean. (c) Peak velocity plotted as a function of added mass averaged across subjects. (d) Average difference in peak velocity between corresponding probe trials in low effort environment and high effort environment $\left(\Delta P V^{\text {probe }}=P V_{\text {low }}^{\text {probe }}-P V_{\text {high }}^{\text {probe }}\right)$ for each subject; bars are arranged in descending order of $\triangle P V$. Most subjects have a mean positive $\triangle P V$ indicating that they move on average faster in the probe trials belonging to the low effort environment. 
(a)

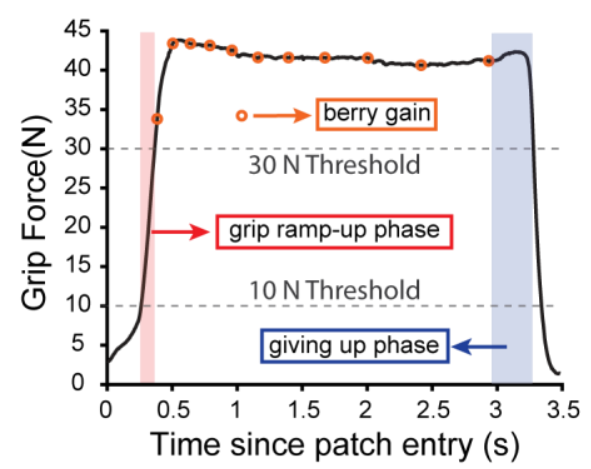

(d)

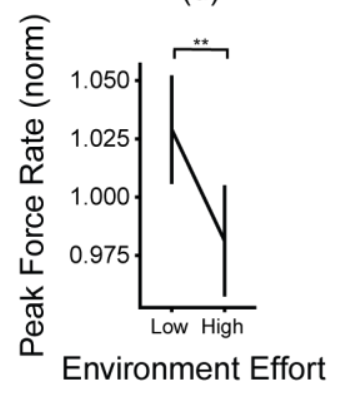

(f)

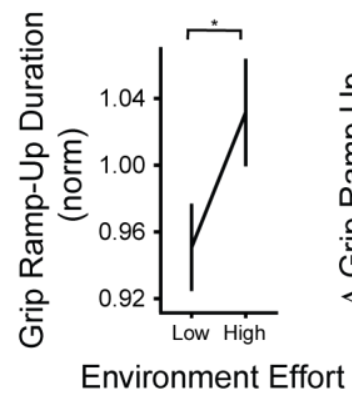

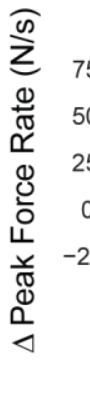

(b)

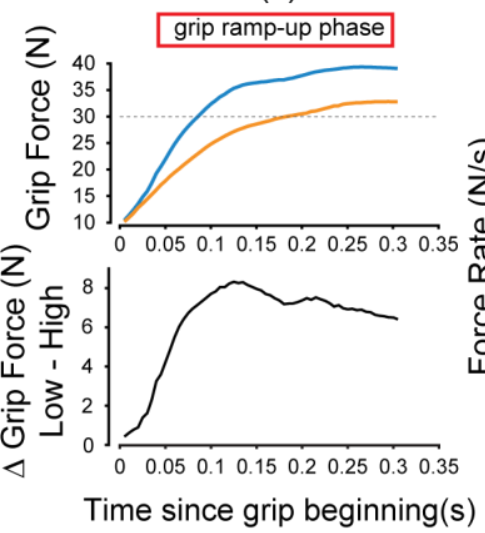

(e)

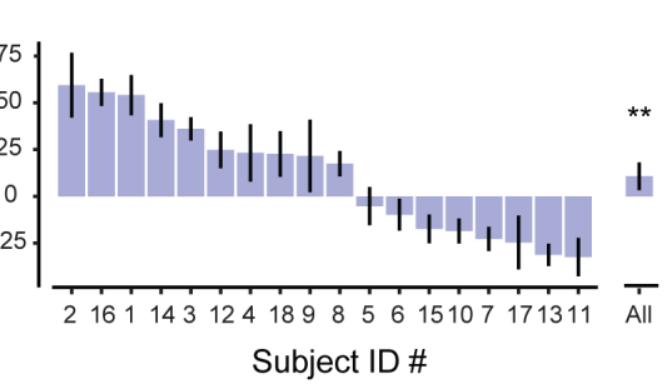

(c)

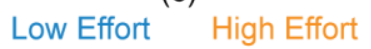

Probe Trials Probe Trials

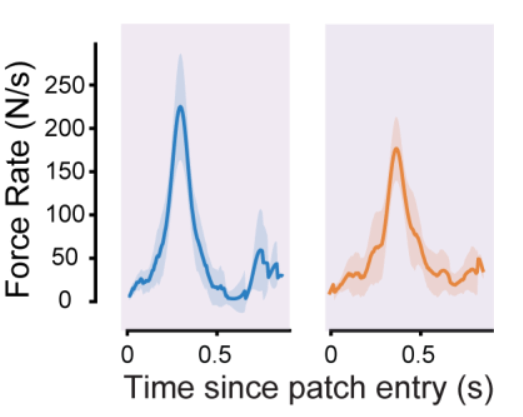

(h)

(g)

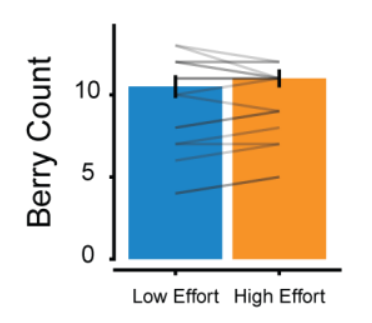

Environment Effort

(i)

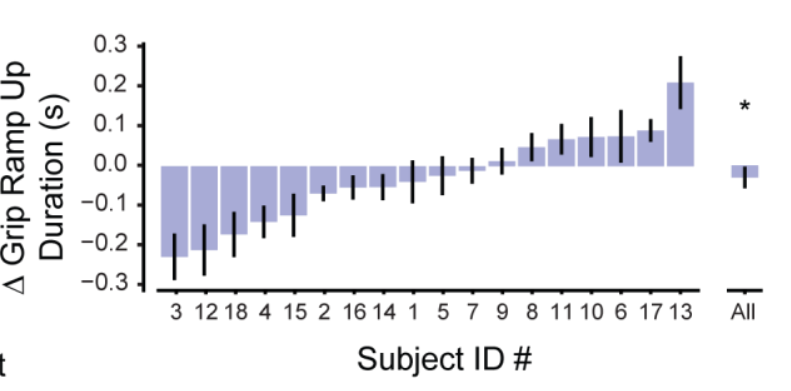

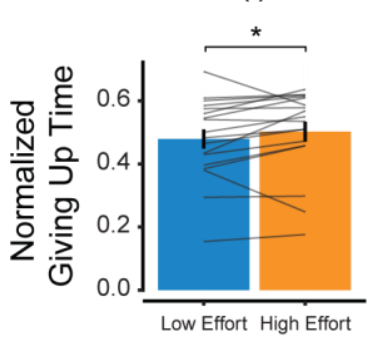

Environment Effort

Figure 5: Harvest behavior is modulated by history of effort (a) A sample grip force profile for an example subject 14 on a randomly selected trial to indicate the temporal decision phases when inside a patch. (b) Zooming in on the grip ramp up phase we compare the grip force profiles between two probe trials for subject 14 , one from the low effort environment (blue) and the other from the high effort environment (orange). (c) Force rate profiles for subject \# averaged across probe trials within the two environments; peak force rate in the probe trials belonging to the low effort environment was higher than that of the high effort environment. (d) Peak force rate - the maximum rate of force generation during ramp-up phase- normalized to subject average, was lower in probe trials belonging to the high effort environment. (e) Most subjects had a positive $\Delta$ Peak force rate. (f) Grip Ramp-up Duration - which is computed as the duration from when the force is at $10 \mathrm{~N}$ to when the force is at $30 \mathrm{~N}$-increased in the probe trials belonging to the high effort environment as opposed to the low effort environment. (g) Mean difference in harvest reaction time in probe trials between environments. As expected, $\Delta$ Harvest reaction time is negative, for most subjects. (h) Berry count modulation by environment was not statistically significant. (i) Giving-up time, normalized to the wait time between the last two berries, increased in the high effort environment. 
During foraging, the decision-making and movements of an individual may be linked via a single normative utility: the sum of rewards acquired, minus efforts expended, divided by time (Shadmehr \& Ahmed, 2020). A prediction of this theory is that the history of reward should affect decision-making during harvesting, and movements during travel. Indeed, we found that people harvested longer and moved slower following a history of low reward (or high effort), and harvested for a shorter duration and moved faster following a history of high reward (or low effort). History of effort had a similar effect on reach vigor: following a history of high effort expenditure, people reduced their reach velocity in probe trials. However, unlike the theoretical predictions, history of high effort did not encourage longer harvest durations. Rather, it slowed the force production patterns that initiated and ended the harvest.

History of reward and effort influenced both the choice of how long to harvest, and the control of movement vigor to the next opportunity. There is a large body of work demonstrating that humans and other animals modulate their harvest duration in patches based on reward history in accordance with MVT (Constantino \& Daw, 2015; Hills et al., 2012; Krebs et al., 1974; Le Heron et al., 2020; Wikenheiser et al., 2013; Wolfe, 2017; Yoon et al., 2018). In contrast, the influence of reward history on movement vigor between patches is considerably less understood. Our observation that movement vigor is also modulate in accordance with MVT indicate that similar processes underlie the control of movement and decision making.

In the context of options immediately available, recent work has revealed a link between movement vigor and preference. For instance, it has been shown that humans (Sackaloo et al., 2015; Summerside et al., 2018) and other animals (Mosberger et al., 2016; Opris et al., 2011) are willing to be energetically inefficient by reaching faster, and even reacting sooner, towards increased reward. This invigoration has been reflected in saccades as well with faster velocities (Haith et al., 2012; Reppert et al., 2015) and shorter reaction times (Kawagoe et al., 1998; Takikawa et al., 2002) observed for higher reward at the end of the movement. Added effort also appears to slow down reaching and walking movements (Gordon et al., 1994; Ralston, 1958; Shadmehr et al., 2016). Moreover, when choosing between options immediately available, movement vigor reveals our underlying subjective valuation. People saccade faster to the option ultimately chosen(Korbisch et al., 2019; Reppert et al., 2015; Yoon et al., 2018). Our contribution in this work is to demonstrate that this connection between movement vigor and decision making is observed not only for immediate reward, but for the history of reward as well. Specifically, we observe a consistent effect of local availability of reward as well as the history of reward on the control of vigor.

In the field of motor control, the history of actions performed has also been shown to play a role in determining the kinematics of subsequent movements. In fact, research has shown that movement repetitions lead to an experience-dependent learning process whereby the brain learns to reduce variability of performance towards previously repeated targets (Diedrichsen et al., 2010; Verstynen \& Sabes, 2011). This has been postulated to be a trial-by-trial learning process of the statistics of the previous actions with a bias-variance tradeoff strategy explaining selected action kinematics in relation to allowed preparation time. This is observed in bias in speed as well as reaction time of repeated movements (Hammerbeck et al., 2014; Mawase et al., 2018). This use-dependent effect is however separable from another history dependent phenomenon observed whereby subjects use a cognitive, predictive strategy when deciding subsequent movements in the presence of uncertainty (Marinovic et al., 2017). But what about the effect of dissimilar actions and their outcomes on subsequent movement kinematics? The optimal foraging framework thus seeks to explain durations of different actions, by defining a unifying ecological utility that the actions and their durations modulate. Our findings demonstrate that both the local context of reward and effort as well as the history of reward and effort significantly affect vigor.

309 Consistent with the predictions of the generalized MVT, people traveled more slowly following a history 310 of high effort. These results are however at odds with those presented by Yoon and colleagues for saccade 
vigor. They found that after history of high effort, imposed by high eccentricity of images, while gaze increased in accordance with the theorem, saccade velocity also increased. In other words, subjects made faster saccades in a poorer environment when moving across equivalent distances between images of identical eccentricity. This result was discussed by the authors as being more aligned with a framework in which effort expenditure elevated the subjective reward value in the environment. This elevation of the reward due to effort expenditure is referred to as justification of effort whereby the same reward higher is valued more if more effort was required to obtain it (Klein et al., 2005; Tricia, S et al., 2000). Though Yoon et al's results contrast with ours, in our experiment 2, we found that some subjects moved faster in the high effort environment as opposed to the low effort environment in the probe trials. The justification of effort phenomenon could explain these inter-individual differences.

In the context of modulated travel effort, we found that some aspects of harvest behavior were modulated by effort history (Figure 5). Specifically, we also find modulation in giving-up duration, a metric that has been used to define how long an animal remains in a patch without reward before leaving. In previous discrete reward foraging scenarios, an increase in giving-up duration has been seen to reflect the forager's perception of environment quality or capture rate. For instance, birds have been known to have a longer giving-up time in poorer environments as opposed to poorer environments (Krebs et al., 1974). As in the literature, and per the prediction of MVT, we find that subjects take longer before giving-up in the high effort environment, aka, the worse environment with the lower opportunity cost.

The neural correlates of reward history remain poorly understood. Dopamine levels in the nucleus accumbens correlate with reward rate, as well as shorter response latencies (Mohebi et al., 2019). However, the source of the increase remains elusive since in that same task, tonic firing rate of dopaminergic neurons do not track the history or reward or punishment (Cohen et al., 2015; Mohebi et al., 2019). A clue to this puzzle may lie in another neurotransmitter: serotonin. Tonic firing rates of serotonergic neurons can reflect history of reward, with other serotonin neurons encoding history of punishment (Cohen et al., 2015). Artificial activation of serotonin neurons leads to increased harvest durations in a foraging task (Lottem et al., 2018), as well as reduced movement vigor (Correia et al., 2017; Seo et al., 2019). Taken together, these results suggest that serotonin may play a role in moderating decision making and movement vigor during foraging via an encoding of reward history.

Not all of our findings conformed with MVT predictions. Previous work has found that after longer travel delays, birds will stay longer and collect more food in subsequent patches with depleting rewards (Cuthill et al., 1990). This has been shown in humans as well, where individuals harvesting reward from virtual trees will collect more apples after a longer travel delay (Constantino \& Daw, 2015). These imposed travel delays seek to reduce the average capture rate of the foraging environment and therefore led to longer stay times. However, in our study we see a lack of modulation of the absolute number of berries collected in response to changing environment quality due to change in travel effort. In our protocol, each berry is associated with a short high-pitched beep, providing a salient signal regarding the number of berries harvested. We believe that subjects settle on several berries that leads to a predictable auditory pattern, that in turn acts as a cue as to when to leave a patch. In other words, subjects learn to expect a certain number of berries irrespective of reward rate due to the auditory feedback, rather than deciding on a per-patch basis. There has been some conflicting evidence for (Mcnair, 1982) and against (Krebs et al., 1974) the phenomenon of predators expecting a set number of prey, or hunting by expectation as opposed to a strategy based on MVT. Nevertheless, in our protocol for experiment 2 we believe that the increased saliency associated with the number of berries primes subjects to collect a fixed number between environments. 354 Notably, in experiment 1 , where auditory feedback was not linked to number of berries, we observed a consistent effect of environment on berries harvested, in accordance with MVT predictions. 
The MVT framework presents an implicit, circular solution (Stephens \& Krebs, 1986) for the optimal durations it prescribes, which are dependent on the very quantity it tries to optimize. This leads to the assumption that the forager has complete knowledge of $J$, on which its corollaries especially rely. Therefore, the theorem is unable to account for transients where the agent needs to learn the quality of the environment as a parameter that can be updated (Mcnamara \& Houston, 1985). In our data, we see that the environment's influence of vigor appears to get washed out by the final block of the environment with the difference between the probe trials' vigor vanishing to zero. Further the theorem does not allow for stochasticity in the environment, as it does not account for fluctuating beliefs of the forager regarding the quality (Oaten, 1977). This has been critiqued in the ecology literature by several studies that have presented alternatives (Green, 1980; Mcnamara \& Houston, 1985; Oaten, 1977; Pyke, 2019). Yet, despite the criticism, empirical studies show that humans and animals consistently behave according to the theorem's predictions. There is now a push to understand the underpinnings of everyday decisions from an ethological standpoint (Hayden, 2018; Mobbs et al., 2018) through more naturalistic experiment designs that better reflect decisions faced by individuals every day. The integration of motor control into the theory is therefore promising in its ability to not just explain motor characteristics, but also evaluation of rewards and costs by investigating corresponding motor decisions.

372 In conclusion, for identical immediate reward and effort opportunities, harvest duration and movement 373 vigor were modulated by the history of reward and effort. People harvested longer and moved slower 374 following a history of low reward, and conversely, harvested for a shorter duration and moved faster 375 following a history of high reward. In accordance with the maximization of common normative utility, 376 history of reward and effort exerted a consistent effect on not only the choice of how long to stay in the 377 current patch but how fast to move to the next one. 


\section{Materials and Methods:}

\section{Theoretical Development:}

Here we describe the hypotheses of the generalized marginal value theorem (MVT) in detail. Mathematically, the marginal capture rate of a patch is represented by $f_{n}\left(t_{h}\right)$ (Eq. 1$) ; r\left(t_{h}\right)$ and $u_{h}\left(t_{h}\right)$ represent the reward obtained in the patch and corresponding costs incurred as a function of harvest duration, respectively. $f_{n}\left(t_{h}\right)$ is also called the harvest function and can be thought of as the net intake from a patch. Additionally, we represent the cost associated with moving to the patch, or movement expenditure, as $u_{m}\left(d, t_{m}^{(n)}\right)$. The average capture rate of the environment is consequently given by $\bar{J}$ (Eq. 2) as the net gain associated with a patch (harvest function minus movement expenditure to patch) divided by the sum of the total time moving to and harvesting from each patch, for all patches.

$$
\begin{aligned}
& f_{n}\left(t_{h}\right)=r\left(t_{h}\right)-u_{h}\left(t_{h}\right) \\
& \bar{J}=\frac{\sum_{n} f_{n}\left(t_{h}^{(n)}\right)-u_{m}\left(d, t_{m}^{(n)}\right)}{\sum_{n} t_{h}^{(n)}+t_{m}^{(n)}}
\end{aligned}
$$

Differentiating $\bar{J}$ in Eq. 2 with respect to $t_{h}$ and $t_{m}$ and equating to zero returns the optimal solutions. $t_{h}^{*}$ is the duration at which the marginal capture rate of a patch equals $\bar{J}$, the average capture rate of the environment. Similarly, $t_{m}^{*}$ is the movement duration for which the movement expenditure rate equals $\bar{J}$ in magnitude. These solutions for optimal harvest duration and movement vigor lead us to two predictions of the theorem:

1. An increase in the effort needed to harvest $\left(u_{h}\right)$ in the patch, or the effort needed to move to the patch $\left(u_{m}\right)$, should produce an increase in harvest duration, and a decrease in movement speed.

2. After a history of high effort, or in expectation of high effort, harvest duration should increase, and movement vigor should decrease.

The normative MVT model used by Yoon et al (Yoon et al., 2018) naturally incorporates experience and subsequent behavior when describing vigor of movements as well as persistence to stay in patch. To test if these predictions hold for arm reaching, we designed a patch foraging task involving reaching movements. The effort of a reach was based on the effort cost model described by (Shadmehr et al., 2016) (Eq 3). In our experiment, we chose to modulate the effort of arm reaches by changing the mass required to be carried during the movement. $m$ was the mass carried during movement, $d$ was the distance travelled and $t_{m}$ was the duration.

$$
u_{m}\left(d, t_{m}\right)=a m t_{m}+b \frac{m d^{2}}{t_{m}}
$$

Further, desire to remain in a patch was fueled by point-based reward that depleted with time spent and was discounted by effort required while harvesting points in a patch. This harvest effort was realized through a force that the subject had to apply through production of grip forces to earn points in a patch. Grip force required in a patch was given by $F_{g}$; so, for harvest duration $t_{h}$, the effort associated with grip force in a patch was given by $u_{h}\left(t_{h}\right)$ (Eq. 4$)$.

$$
u_{h}\left(t_{h}\right)=F_{g} * t_{h}
$$

To test the predictions of the theory, we designed a foraging protocol in which subjects collected rewards in two environments, one with high effort, and the other with low effort (Figure 2). Subjects reached between patches. Once in a patch, they produced a grip force to harvest reward. In one environment, most 
trials had no added mass (Eq. 3; $m=0$ ), while in the second environment most trials had high added mass $(m=3.5 \mathrm{~kg})$. In both environments, they experienced some trials with intermediate added mass $(m=$ $2 \mathrm{~kg})$. All patches required a constant grip force $\left(F_{g}=30 \mathrm{~N}\right)$.

For our experiment design, we have the following predictions based on our hypotheses:

H1. Subjects will move faster in the probe trials belonging to the better environment (high reward or low effort), as compared to the poorer environment.

H2. Similarly, we expect that subjects will shorten their harvest duration in the better environment (high reward or low effort), as compared to the poorer environment. Specifically, we compare the equivalent probe trials between the two environments to isolate the effects of reward and effort history in the two experiments.

\section{Experiment Protocol and Design:}

Subjects: Thirty-four subjects (age $=25.5 \pm 3.5$ years, 15 female) participated in the study, fourteen in experiment 1 and twenty in experiment 2. All subjects were healthy with no recent injuries or known pathologies. Consent was obtained in accordance with the University of Colorado Institutional Review Board. Subjects were paid a base amount of $\$ 10$ an hour, but their final compensation depended on their performance. Two subjects were unable to complete the experiment due to their selection of very long harvest duration in experiment 2 and therefore results for this session presented are from the 18 subjects who completed all the trials in the experiment.

Apparatus and Data Acquisition: To test predictions of MVT in humans, a foraging task involving arm reaching movements was designed. Subjects were seated in a chair with full back support and made horizontal, planar reaches while grasping the handle of a robotic manipulandum (InMotion 2; Interactive Motion Technologies, Shoulder-elbow robot 2) as seen in Figure 2a. By moving the robot handle subjects controlled the movement of a cursor on the monitor placed at eye-level. The monitor displayed a game screen in which they were cued to move to different targets, causing them to make reaches in different directions in the horizontal plane. The end of the robot handle was attached with a grasp sensor that measured force with which subjects gripped the handle. Additionally, the robot could produce forces; here we leveraged this by having the robot produce acceleration-dependent resistive forces to simulate the effect of adding mass to the reach in the horizontal plane for experiment 2 (Eq. 5). Robot forces were inactive during experiment 1 .

$$
\left[F_{x} F_{y}\right]=-1 *\left[\begin{array}{cc}
m & 0 \\
0 & m
\end{array}\right] *\left[a_{x} a_{y}\right]
$$

Reach position, velocity, acceleration, and corresponding grip force of every trial was recorded by the robot at a frequency of $200 \mathrm{~Hz}$. The force field produced by robot motors during a reach was also continuously updated, based on the current acceleration as recorded by the accelerometer, at a rate of $200 \mathrm{~Hz}$.

Experiment 1: This experiment emulated a patch foraging task in which subjects could move between patches by making reaching movements with their dominant arm (Figure 2a). On the screen in front of them the foraging task appeared; subjects were instructed to move their cursor into a circular patch of reward on the screen (Figure 2b). Once their cursor was in the patch, they could collect reward by making pulsed grip forces (Figure 2c). Subjects had to wait for the patch to turn green upon which they were required to hit a threshold force of $30 \mathrm{~N}$ and return their force back to under $10 \mathrm{~N}$, at which point they received rewarding 'berries'. Subjects could wait for the patch to turn green to collect more reward or move on to the next at 
any time. Their total earnings from a patch were added to a cumulative score counter at the top of the screen. An example grip force profile is seen in Figure 2e.

Overall, subjects experienced three patch types with differing reward, each indicated by a different patch color (Figure 2d). Reward was dispensed per grip according to an exponential decay function, jittered by gaussian noise $r(n) \sim N\left(\beta^{n-1} \alpha, 1\right)$, where $\alpha$ represents the maximum reward that can be obtained from a pulse, and $\beta$ represents the decay rate fixed at 0.8 for the entirety of this experiment. the first pulse always resulted in the maximum reward, $r(1)=\alpha$ (Figure $2 \mathrm{f}$ ).

Before the main experiment, subjects were familiarized with the patch types with 30 trials, 10 for each reward value. Subjects were questioned about which patch was the most rewarding to ensure they had internalized the color-reward mapping. If they responded incorrectly, they were re-familiarized. No subject required more than 2 familiarization sessions; familiarization data were not included in any analyses presented.

Experiment 2: In this experiment, the effort of travel was modulated by adding mass to the reaches between the patches. A brief familiarization session was conducted to acquaint subjects to the passive inertial forces of the robot arm as well as the acceleration-dependent force-field. Subjects were then required to collect berries in a task similar to experiment 1 . The available patch was displayed by a red circle. Subjects moved the robot handle and placed a cursor inside the patch. Once inside the patch, they produced a grip force that was specified by an indicator next to the patch circle (Figure $2 \mathrm{~g}$ ). Harvesting began when the grip force reached the minimum required force. Berry harvesting was indicated by an animation: an orange circle quickly appearing and disappearing, accompanied by a high-pitched beep. Berries were harvested at a declining rate while the grip force was maintained above the minimum level. The total number of berries collected over a duration $t_{h}$ was specified by the function $r\left(t_{h}\right)=\alpha\left(1-\frac{1}{1+\beta t_{h}}\right)$. Essentially, the time between successive berries at any given instance was calculated from the instantaneous reward rate resulting in consecutive berries having longer durations between them as time spent in the patch increased. Here, $\alpha$ represents the maximum reward and $\beta$ represents rate of decline in reward harvested. Subjects were instructed to hold their force at the required level and reduce it only when they intended to move out of the patch, i.e., they were discouraged from 'resting' inside the patch. They were told that they were free to leave the current patch at any point in time towards the cued position where a new patch would appear. Once they exited a patch, it disappeared, and a new replenished patch appeared in the new location (Figure $2 \mathrm{~g}$ ). Patches always appeared at the same two positions thereby keeping the travel distance constant at $d=$ $30 \mathrm{~cm}$ across the entire experiment. Subjects $(\mathrm{n}=18)$ experienced the foraging task in two main blocks of trials: low mass and high mass environments. Travel effort was modulated by changing the added mass $m$ to the reach period and harvest effort was represented by the amount of grip force that was to be maintained to ensure berry "consumption".

Subjects experienced three effort levels with three different values of added mass. This was cued to the subject by means of cursor appearance (Figure $2 \mathrm{~h}$ ). The protocol entailed foraging in two environments. The low effort environment entailed no added mass $(m=0 \mathrm{~kg})$ for most trials. The high effort environment entailed large added mass $(m=3.5 \mathrm{~kg})$. Both environments had probe trials in which the added mass was $m=2 \mathrm{~kg}$. Each environment contained two hundred trials that was divided into four sub-blocks of fifty trials each, not including 30 trials in which subjects were familiarized with the parameters of the environment. Subjects were not informed of the total number of trials in each environment. Rather, they were told that the total session duration was about one hour and fifteen minutes, including instruction and consent procedures. Within each sub-block of fifty, the middle ten trials were designated as probe thereby leading to forty total probe trials in each environment. There was no discernable break between trials in the 
two environments. For all trials in both environments, including probe trials, the minimum amount of grip force required to harvest berries within each patch was fixed $\left(F_{g} \geq 30 N\right)$.

When travelling between the two patches, the amount of added mass was indicated by means of a modified cursor. Subjects were also instructed not to increase the grip force during travel between patches, to decouple harvest and movement efforts. If they chose to travel with increased grip force $(>10 \mathrm{~N})$, the game paused, a message appeared on screen asking them to reduce their grip force. Subjects were also informed that every 10 berries collected would correspond to $1 \varnothing$ in monetary bonus, to increase task engagement.

In both experiments, environment order was counterbalanced across subjects.

Behavioral metrics of movement and harvest: In experiment 1, our primary hypothesis concerns the modulation of movement vigor by changing the history of reward. Since movement is realized through reaches between patches, we compute the maximum velocity in the horizontal direction over the time course of the reach and use it to quantify the vigor of the movement. The horizontal velocity is chosen because the primary movement happens in that direction by virtue of the position of all the patches being along the horizontal axis. Therefore, vigor is quantified through peak velocity of reaching.

For harvest behavior, we use two metrics. One is the absolute number of berries harvested per patch to denote harvest behavior in the patch. The next metric is the absolute duration of harvest within a patch since patch entry to patch exit in seconds. Therefore, harvest behavior is quantified by berries harvested as well as harvest duration.

For experiment 2, our primary hypothesis here is concerned with how movement vigor is modulated by changing the history of effort. As in the previous experiment, the horizontal velocity is chosen because the primary movement happens in that direction by virtue of the position of all the patches being along the horizontal axis.

We quantified behavior using four metrics: harvest reaction time, peak force rate, number of berries, and giving-up time. Rate of force was computed as the numerical derivative of force over the course of the trial which was then filtered through a low-pass Butterworth filter with a $10 \mathrm{~Hz}$ cut-off frequency. Since this was a grip-and-hold task. The harvest reaction time was defined as the duration from when subjects increase grip force above $10 \mathrm{~N}$ until the point at which they reach the required threshold of $30 \mathrm{~N}$. Peak force rate was the maximum rate of force generation during the grip ramp-up period. Together, harvest reaction time and peak force rate determined the speed with which the subjects began harvest following arrival in the patch. The number of berries per patch was computed as the total number of berries in a patch that subjects collect. Finally, we computed giving-up time as the duration after the last berry was collected until they reduced the grip force below the minimum. Because berries were dispensed after increasingly long intervals, this quantity was normalized to the duration between the second to last and last berries collected.

Statistical Analyses: Therefore, based on our design, each environment had two trial types- exemplar trials (representing the actual attributes of the environment) and probe trials (different from exemplar but equivalent across environments). MVT predicts an effect of immediate reward and effort, i.e., if reward increases, or effort decreases, it should equivalently affect vigor as well as harvest duration. This was tested by comparing exemplar trials across both environments. The critical test for MVT in this case comes from the comparison of probe trials between the two environments. We therefore combined all the trials across each environment and obtained average behavior across the block of fifty trials. This captures temporal behavior as well differences between environments. We performed a two-way repeated measures ANOVA on each trial type to obtain the effects of environment as well as the effect of trail number within block. 
The two equations representing these ANOVAs for each trial type are presented (Equations $9 \& 10$ ). Met represents the above metrics for movement vigor as well as harvest duration.

$$
\begin{gathered}
\text { Met }_{\text {exemplar }} \sim \text { Env }+ \text { trialInBlock }+\operatorname{Error}(\operatorname{Subj}) \#(9) \\
\text { Met }_{\text {probe }} \sim \text { Env }+ \text { trialInBlock }+\operatorname{Error}(\operatorname{Subj}) \#(10)
\end{gathered}
$$

We also completed 6 post-hoc t-tests based on observing the data to test the effect of the transitioning between exemplar and probe trials for each metric. This helped us determine if the sudden changes in harvest duration and peak velocity before the transition compared to neighboring trials of the same trial type were statistically significant. For harvest duration we compared trials 29 and 30 and trials 30 and 31 in the block average for each subject in the low reward environment. Correspondingly in the high reward environment we compared trials 19 and 20 as well as trials 20 and 21. For peak velocity in the low reward environment we compared trials 30 and 31 as well as trials 31 and 32. And for the high reward environment we compared trials 20 to 21 and trials 21 to 22 within the block averages for each subject.

We use a statistical threshold of $\alpha=0.05$ for all comparisons. P-values are reported exactly unless they are less than 0.001 in which they are reported as such. All the F-statistics, confidence intervals and p-values are reported in the results for each metric.

\section{References:}

Charnov, E. L. (1976). Optimal Foraging, the Marginal Value Theorem. 136, 129-136.

Cohen, J. Y., Amoroso, M. W., \& Uchida, N. (2015). Serotonergic neurons signal reward and punishment on multiple timescales. ELife, 2015(4), 1-25. https://doi.org/10.7554/eLife.06346

Constantino, S. M., \& Daw, N. D. (2015). Learning the opportunity cost of time in a patch-foraging task. Cognitive, Affective, \& Behavioral Neuroscience, 15, 837-853. https://doi.org/10.3758/s13415-0150350-y

Correia, P. A., Lottem, E., Banerjee, D., Machado, A. S., Carey, M. R., \& Mainen, Z. F. (2017). Transient inhibition and long-term facilitation of locomotion by phasic optogenetic activation of serotonin neurons. ELife, 6, 1-27. https://doi.org/10.7554/eLife.20975

Cowie, R. J. (1977). Optimal foraging in great tits (Parus Major). Nature, 268(July), 137-139. https://doi.org/https://doi.org/10.1038/268137a0

Cuthill, I. C., Kacelnik, A., Krebs, J. R., Haccou, P., \& Iwasa, Y. (1990). Starlings exploiting patches: the effect of recent experience on foraging decisions. Animal Behaviour, 40(4), 625-640. https://doi.org/10.1016/S0003-3472(05)80692-X

Diedrichsen, J., White, O., Newman, D., \& Lally, N. (2010). Use-Dependent and Error-Based Learning of Motor. Journal of Neuroscience, 30(15), 5159-5166. https://doi.org/10.1523/JNEUROSCI.540609.2010

Gordon, J., Ghilardi, M. F., Cooper, S. E., \& Ghez, C. (1994). Accuracy of planar reaching movements II. Systematic extent errors resulting from inertial anisotropy. Experimental Brain Research, 99(1), 112-130. https://doi.org/10.1007/BF00241416

Green, R. F. (1980). Bayesian Birds: A Simple Example of Oaten's Stochastic Model of Optimal Foraging. Theoretical Population Biology, 244-256. 
Haith, A. M., Reppert, T. R., \& Shadmehr, R. (2012). Evidence for Hyperbolic Temporal Discounting of Reward in Control of Movements. Journal of Neuroscience. https://doi.org/10.1523/JNEUROSCI.0424-12.2012

Hammerbeck, U., Yousif, N., Greenwood, R., Rothwell, J. C., \& Diedrichsen, J. (2014). Movement speed is biased by prior experience. Journal of Neurophysiology, 111(1), 128-134. https://doi.org/10.1152/jn.00522.2013

Hayden, B. Y. (2018). Economic choice : the foraging perspective. 1-6. https://doi.org/https://doi.org/10.1016/j.cobeha.2017.12.002

Hayden, B. Y., Pearson, J. M., \& Platt, M. L. (2011). Neuronal basis of sequential foraging decisions in a patchy environment. Nature Neuroscience, 14(7), 933-939. https://doi.org/10.1038/nn.2856

Hills, T. T., Jones, M. N., \& Todd, P. M. (2012). Optimal foraging in semantic memory. Psychological Review, 119(2), 431-440. https://doi.org/10.1037/a0027373

Kawagoe, R., Takikawa, Y., \& Hikosaka, O. (1998). Expectation of reward modulates cognitive signals in the basal ganglia. Nature Neuroscience, 1(5), 411-416. https://doi.org/10.1038/1625

Klein, E. D., Bhatt, R. S., \& Zentall, T. R. (2005). Contrast and the justification of effort. Psychonomic Bulletin and Review, 12(2), 335-339. https://doi.org/10.3758/BF03196381

Korbisch, C., Apuan, D., \& Ahmed, A. A. (2019). Saccade vigor reveals relative utility in effort-based choice. Motor Learning and Motor Control Symposium.

Krebs, J. R., Ryan, J. C., \& Charnov, E. L. (1974). Hunting by expectation or optimal foraging? A study of patch use by chickadees. Animal Behaviour, 22(PART 4), 953-964. https://doi.org/10.1016/00033472(74)90018-9

Le Heron, C., Kolling, N., Plant, O., Kienast, A., Janska, R., Ang, Y. S., Fallon, S., Husain, M., \& Apps, M. A. J. (2020). Dopamine Modulates Dynamic Decision-Making during Foraging. Journal of Neuroscience, 40(27), 5273-5282. https://doi.org/10.1523/JNEUROSCI.2586-19.2020

Lottem, E., Banerjee, D., Vertechi, P., Sarra, D., Lohuis, M. O., \& Mainen, Z. F. (2018). Activation of serotonin neurons promotes active persistence in a probabilistic foraging task. Nature Communications, 9(1), 1-12. https://doi.org/10.1038/s41467-018-03438-y

Marinovic, W., Poh, E., De Rugy, A., \& Carroll, T. J. (2017). Action history influences subsequent movement via two distinct processes. ELife, 6, 1-23. https://doi.org/10.7554/eLife.26713

Mawase, F., Lopez, D., Celnik, P. A., \& Haith, A. M. (2018). Movement Repetition Facilitates Response Preparation. CellReports, 24(4), 801-808. https://doi.org/10.1016/j.celrep.2018.06.097

Mcnair, J. N. (1982). Optimal giving-up times and the marginal value theorem. The American Naturalist, 119(4), 511-529. https://doi.org/https://doi.org/10.1086/283929

Mcnamara, J. M., \& Houston, A. I. (1985). Optimal Foraging and Learning. Journal of Theoretical Biology, 117, 231-249. https://doi.org/https://doi.org/10.1016/S0022-5193(85)80219-8

Mobbs, D., Trimmer, P. C., Blumstein, D. T., \& Dayan, P. (2018). Foraging for foundations in decision neuroscience : insights from ethology. https://doi.org/10.1038/s41583-018-0010-7

Mohebi, A., Pettibone, J. R., Hamid, A. A., Wong, J. M. T., Vinson, L. T., Patriarchi, T., Tian, L., Kennedy, R. T., \& Berke, J. D. (2019). Dissociable dopamine dynamics for learning and motivation. Nature, 570(7759), 65-70. https://doi.org/10.1038/s41586-019-1235-y 
Mosberger, A. C., De Clauser, L., Kasper, H., \& Schwab, M. E. (2016). Motivational state, reward value, and Pavlovian cues differentially affect skilled forelimb grasping in rats. Learning and Memory, 23(6), 289-302. https://doi.org/10.1101/lm.039537.115

Niv, Y., Daw, N. D., Joel, D., \& Dayan, P. (2007). Tonic dopamine: Opportunity costs and the control of response vigor. Psychopharmacology. https://doi.org/10.1007/s00213-006-0502-4

Oaten, A. (1977). Optimal foraging in patches: A case for stochasticity. Theoretical Population Biology, 12(3), 263-285. https://doi.org/10.1016/0040-5809(77)90046-6

Opris, I., Lebedev, M., \& Nelson, R. J. (2011). Motor planning under unpredictable reward: Modulations of movement vigor and primate striatum activity. Frontiers in Neuroscience, 5(MAY), 1-12. https://doi.org/10.3389/fnins.2011.00061

Perry, C. J., Baciadonna, L., \& Chittka, L. (2016). Unexpected rewards induce dopamine-dependent positive emotion-like state changes in bumblebees. Science, 353(6307), 1529-1532. https://doi.org/10.1126/science.aaf4454

Pyke, G. H. . (2019). Optimal Foraging Theory : A Critical Review. Annual Review of Ecology and Systematics, 15(1984), 523-575. https://www.jstor.org/stable/2096959

Ralston, H. J. (1958). Energy-speed relation and optimal speed during level walking. Internationale Zeitschrift Für Angewandte Physiologie Einschliesslich Arbeitsphysiologie, 17(4), 277-283. https://doi.org/10.1007/BF00698754

Reppert, T. R., Lempert, K. M., Glimcher, P. W., \& Shadmehr, R. (2015). Modulation of saccade vigor during value-based decision making. Journal of Neuroscience, 35(46), 15369-15378. https://doi.org/10.1523/JNEUROSCI.2621-15.2015

Richardson, H., \& Verbeek, N. A. M. (1987). Diet selection by yearling northwestern crows (Corvus caurinus) feeding on littleneck clams (Venerupis japonica). Auk, 104(2), 263-269. https://doi.org/10.1093/auk/104.2.263

Rigoux, L., \& Guigon, E. (2012). A Model of Reward- and Effort-Based Optimal Decision Making and Motor Control. 8(10). https://doi.org/10.1371/journal.pcbi.1002716

Sackaloo, K., Strouse, E., \& Rice, M. S. (2015). Degree of preference and its influence on motor control when reaching for most preferred, neutrally preferred, and least preferred candy. OTJR Occupation, Participation and Health, 35(2), 81-88. https://doi.org/10.1177/1539449214561763

Schweighofer, N., Xiao, Y., Kim, S., Yoshioka, T., Gordon, J., \& Osu, R. (2015). Effort, success, and nonuse determine arm choice. Journal of Neurophysiology, 114(1), 551-559. https://doi.org/10.1152/jn.00593.2014

Seo, C., Guru, A., Jin, M., Ito, B., Sleezer, B. J., Ho, Y. Y., Wang, E., Boada, C., Krupa, N. A., Kullakanda, D. S., Shen, C. X., \& Warden, M. R. (2019). Intense threat switches dorsal raphe serotonin neurons to a paradoxical operational mode. Science, 363(6426), 539-542. https://doi.org/10.1126/science.aau8722

Shadmehr, R., \& Ahmed, A. A. (2020). Vigor: Neuroeconomics of movement control. MIT Press.

Shadmehr, R., Huang, H. J., \& Ahmed, A. A. (2016). A Representation of Effort in Decision-Making and Motor Control. Current Biology, 26, 1-6. https://doi.org/10.1016/j.cub.2016.05.065

Stephens, D. W., \& Krebs, J. R. (1986). Foraging Theory. In Foraging Theory. Princeton University Press. https://doi.org/10.2307/j.ctvs32s6b 
Summerside, E. M., Shadmehr, R., \& Ahmed, A. A. (2018). Vigor of reaching movements: Reward discounts the cost of effort. Journal of Neurophysiology, 119(6), 2347-2357. https://doi.org/10.1152/JN.00872.2017

Takikawa, Y., Kawagoe, R., Itoh, H., Nakahara, H., \& Hikosaka, O. (2002). Modulation of saccadic eye movements by predicted reward outcome. Experimental Brain Research, 142(2), 284-291. https://doi.org/10.1007/s00221-001-0928-1

Thura, D., Cos, I., Trung, J., \& Cisek, P. (2014). Context-dependent urgency influences speed-accuracy trade-offs in decision-making and movement execution. Journal of Neuroscience, 34(49), 1644216454. https://doi.org/10.1523/JNEUROSCI.0162-14.2014

Tricia, S, C., Feltus, J. R., Daren, H., \& Zentall, T. R. (2000). "Work Ethic" in Pigeons: Reward value is directly related to the effort or time required to obtain the reward. Psychonomic Bulletin and Review, $7(1), 100-106$.

Verstynen, T., \& Sabes, P. N. (2011). How Each Movement Changes the Next : An Experimental and Theoretical Study of Fast Adaptive Priors in Reaching. Journal of Neuroscience, 31(27), 1005010059. https://doi.org/10.1523/JNEUROSCI.6525-10.2011

Wang, A. Y., Miura, K., \& Uchida, N. (2013). The dorsomedial striatum encodes net expected return, critical for energizing performance vigor. Nature Neuroscience, 16(5), 639-647. https://doi.org/10.1038/nn.3377

Wang, J., Lum, P. S., Shadmehr, R., \& Lee, S. W. (2021). Perceived effort affects choice of limb and reaction time of movements. Journal of Neurophysiology, 125(1), 63-73. https://doi.org/10.1152/JN.00404.2020

Wikenheiser, A. M., Stephens, D. W., \& Redish, A. D. (2013). Subjective costs drive overly patient foraging strategies in rats on an intertemporal foraging task. 110(20). https://doi.org/10.1073/pnas.1220738110

Wolfe, J. M. (2017). When is it time to move to the next raspberry bush? Foraging rules in human visual search. 13(2013), 1-17. https://doi.org/10.1167/13.3.10.doi

Yoon, T., Geary, R. B., Ahmed, A. A., \& Shadmehr, R. (2018). Control of movement vigor and decision making during foraging. Proceedings of the National Academy of Sciences, 115(44), E10476 LPE10485. https://doi.org/10.1073/pnas.1812979115 